\title{
RGB Color Distribution Analysis Using Volumetric Fractal Dimension
}

\author{
Dalcimar Casanova* and Odemir Martinez Bruno \\ USP - Universidade de São Paulo \\ IFSC - Instituto de Física de São Carlos, São Carlos, Brasil \\ dalcimar@gmail.com, bruno@ifsc.usp.br
}

\begin{abstract}
Over the years many approaches for texture analysis have been proposed. Most of these methods use, directly or indirectly, the spatial information to build the features. Although the spatial distribution of gray levels is a property a priori of the texture, some methods do not use this propriety to characterize it. The problem is that this class of methods has, generally, worst results than first one. Thus, in this work we propose a new method to classify color textures that does not use any type of spatial distribution information and still achieves high classification rates, comparable, if not better, than traditional texture analysis methods. The method is based on analysis of RGB color distribution using volumetric fractal dimension.
\end{abstract}

\section{Introduction}

The identification of visual patterns in images or objects is a key process in computer vision area. And, among the set of possible patterns, the texture is one of the most useful for experiments of image classification and identification.

Although there is no precise definition of texture, this attribute is easily perceived by humans being a rich source of visual information. However, while the ability of a human to distinguish different textures is apparent, the automated description and recognition of these same patterns has proved to be quite complex.

Many methods of texture analysis have been proposed recently, most of these methods use, directly or indirectly, the spatial distribution of gray levels to build they features. In statistical approaches, the greatest number of methods uses any information of the likelihood of neighboring pixel values (e.g. GLDM[18], GLCM [4]). The geometrical-based methods have an desirable property in defining local spatial neighborhoods (e.g. Voronoi tessellation features [15]. In modelbased methods, such MRF[2], assume that the intensity at each pixel in the image depends on the intensities of only the neighboring pixels. And in signal processing methods, the frequency of an texture is determined by spatial distribution of the pixels [13]).

Although the spatial distribution of gray levels is a property a priori of the texture, some methods do not use this propriety to characterize it. First-order

\footnotetext{
* Dalcimar Casanova gratefully acknowledges the financial support FAPESP (São
} Paulo Research Foundation, Brazil) (2008/57313-2) for his PhD grant. 
statistics, for example, measure the likelihood of observing a gray value at a randomly chosen location in the image. First-order statistics can be computed from the histogram of pixel intensities in the image. These depend only on individual pixel values and not on the interaction or co-occurrence of neighboring pixel values. The problem is that this class of methods are generally, not competitive against the methods that use some spatial distribution information.

So, in this work, we propose a new method that does not use any type of spatial distribution information to classify color textures. They are based on the color distribution analysis over RGB color model. This analysis is made with volumetric fractal dimension and posterior classification with LDA and Bayesian classifier. The results are best than methods of same class and very competitive against another methods with neighborhood relationship.

The rest of the paper is organized as follows. Section 2 presents the general methodology for RGB color cube transform, the volumetric fractal dimension and the classification procedure. Section 3 describes the experimental results and the comparison with others methods, while conclusions are presented in Section 4.

\section{Materials and Methods}

\subsection{RGB Color Cube Transform}

The first step of the proposed method is mapping the existing colors of texture in a cube represented by RGB coordinates (i.e. red, green and blue colors). Given an texture image $I(x, y)$, an cube $C(r, g, b)$ (that have the r-axis representing red values, g-axis as green values and b-axis as blue values), and the colors in $I$ defined by three components, the $C(r, g, b)$ will be an function as follows:

$$
C(r, g, b)=\left\{\begin{array}{l}
1, \text { if } \exists I(x, y)=(r, g, b) \\
0, \text { otherwise }
\end{array}\right.
$$

In proposed method we use each axis in the range 0 to 255 , representing 8 -bit per channel. The basic idea of this transformation is summarize all existent colors in texture and map that in a cube, as is done in 3D color histogram representation, but without counting the number of image pixels in each bin. In Fig. 11 we show 3 different textures that represent this transformation. Note that the spacial distribution of the colors is different for each class. In the next session we will explore and quantify this propriety by use of volumetric fractal dimension.

\subsection{Volumetric Fractal Dimension}

Benoit Mandelbrot, in 1970s, introduced a new field of mathematics, named Fractal Geometry. He said that complex objects are generated by the interaction of simple rules and has non-integer dimension, which is related to its complexity. Since then many methods have been developed to estimate the fractal dimension of an given object, one of the most accurate is the Bouligand-Minkowski 14. 

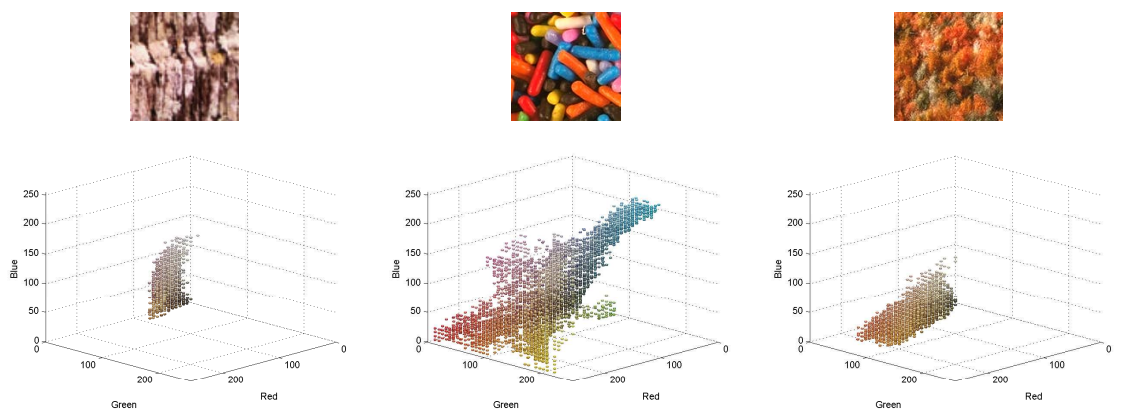

Fig. 1. Textures examples (above) and your respective RGB cube transforms (below). The main idea is summarize all existent colors in texture in a cube that represent RGB coordinates.

Since then some works of texture analysis has been made with fractals [7]. Recently [1] proposed a new texture descriptor based on fractals. In this, the texture is mapped to a cube, and the descriptor explores the differences in influence area of the 3D object formed. The same process will be used here to characterize the spacial distribution of the colors in our RGB color cube transform.

Given our 3D cube $C(r, g, b)$, we can obtain $V(r)$ through dilation of each point $p$ of $C$ using a sphere of radius $r$. This $V(r)$ is the influence volume of the object for a given radius $r$, and is very sensitive to structural changes of the object. As we have different objects, with different structures in your RGB color cube transform, this methodology is suitable to characterize it. The dilation curve expressed as volume $V(r)$ as a function of the dilation radius $r_{\max }$ is given by:

$$
V(r)=\left\{p \in R^{3}\left|\exists p^{\prime} \in S:\right| p-p^{\prime} \mid \leq r\right\}
$$

In this method the arrangement of points in $C$ alters the process of dilation. As the value of $r$ grows, the spheres produced by the different points of object begin to interact. This interaction causes effects in $V(r)$, thus each object produces an characteristic growth of $V(r)$ and this makes possible the use of the values of $V(r)$ as descriptors. Thus, the feature vector $\mathbf{x}$ is defined as the set of logarithm of influence volumes $V(r)$ calculated for all values of $r \in E$, where $E$ is the set of possible Euclidean distances for a radius $r_{\max }$ :

$$
\begin{gathered}
E=\left\{1, \sqrt{2}, \sqrt{3}, \ldots, r_{\max }\right\} \\
\mathbf{x}=\left[\log V(1), \log V(\sqrt{2}), \ldots, \log V\left(r_{\max }\right)\right]
\end{gathered}
$$

The Fig. 2 exemplifies this process of dilation for different values of $r$. In order to complete the estimation of Bouligand-Minkowski fractal dimension we need plot the $\log V(r)$ versus $\log r$. The value of inclination of the straight line obtained gives us an estimative of the fractal dimension of the respective object (Equation 5 ). 

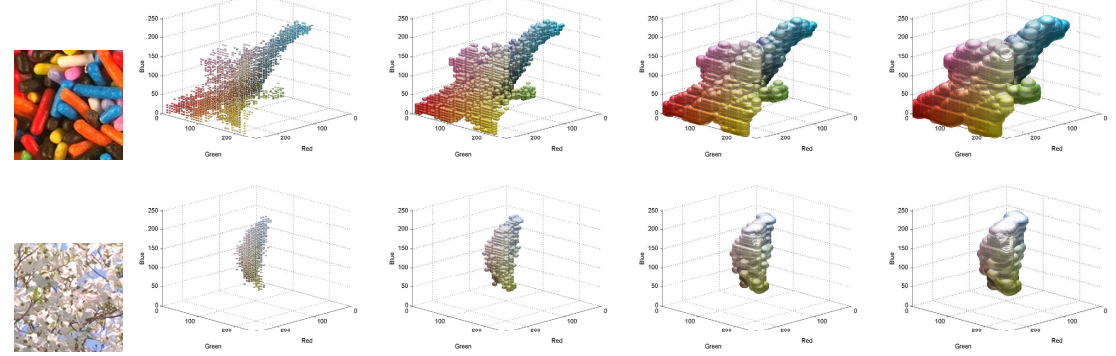

Fig. 2. Dilation process for two different textures. This allows an analysis of color distribution by the volume calculated.

$$
D=3-\lim _{r \rightarrow 0} \frac{\log V(r)}{\log (r)}
$$

The $V(r)$ can be calculated by using some fast Exact Distance Transform (EDT) algorithms [5, 9, 3]. A important characteristic is that only one parameter need be chosen, the $r_{\max }$.

\subsection{Data Analysis}

A widely type of techniques are used for data analysis in a supervised multiclass classification task. Due the values of $V(r)$ are naturally dependent and highly correlated we opt by use the Linear Discriminant Analysis (LDA)+Bayesian classifier. This supervised task is performed under a 10-fold cross-validation scheme.

Linear Discriminant Analysis. Basically, LDA applies an geometric transformation (rotations) to the feature space with the purpose of generating new uncorrelated features based on linear combinations of the original ones, aiming seek a projection that best separates the data. Given the matrix $S$, indicating the total dispersion among the feature vectors, defined as:

$$
S=\sum_{i=1}^{N}\left(\mathbf{x}_{i}-\mu\right)\left(\mathbf{x}_{i}-\mu\right)^{\prime}
$$

and the matrix $S_{i}$ indicating the dispersion of objects of $C_{i}$ :

$$
S_{i}=\sum_{i \in C_{i}}\left(\mathbf{x}_{i}-\mu_{i}\right)\left(\mathbf{x}_{i}-\mu_{i}\right)^{\prime}
$$

we can define the intra-class variability $S_{\text {intra }}$ (indicating the combined dispersion within each class) and interclass variability $S_{\text {inter }}$ (indicating the dispersion of the classes in terms of their centroids) as:

$$
S_{\text {intra }}=\sum_{i=1}^{K} S_{i}
$$




$$
S_{\text {inter }}=\sum_{i=1}^{K} N_{i}\left(\mu_{i}-\mu\right)\left(\mu_{i}-\mu\right)^{\prime}
$$

where $K$ is the number of classes, $N$, the number of samples, $N_{i}$, the number of objects in class $i, C_{i}$, the set of samples of class $i, \mu$, the global average, and $\mu_{i}$, the average of objects in class $i$. For these measures of dispersion we have necessarily:

$$
S=S_{\text {intra }}+S_{\text {inter }}
$$

Thus, the i-th canonical discriminant function is given by:

$$
Z_{i}=a_{i 1} \mathbf{X}_{1}+a_{i 2} \mathbf{X}_{2}+\cdots+a_{i p} \mathbf{X}_{p}
$$

where $p$ is the number of features of the model and $a_{i} j$ are the elements of the eigenvector $a_{i}=\left(a_{i 1}, a_{i 2}, \ldots, a_{i p}\right)$ of matrix $C$ given by:

$$
C=S_{\text {inter }} * S_{\text {intra }}^{-1}
$$

This formulation leads to a condition where there is no correlation between $Z_{i}$ and $Z_{1}, Z_{2}, \ldots$, within the classes. From $p$-original variables the $p$-principal components can be obtained. However, in general, a reduction in the number of variables to be assessed is desired, i.e., the information contained in the p-original variables be replaced by the information contained in $k(k<p)$ uncorrelated principal components. Thus, the system of random variability of the original vector with $p$-original variables is approximated by the variability of the random vector containing the $k$-principal components.

Bayesian classifier. The Bayesian classifier is based on the Bayesian decision theory and combines class conditional probability densities (likelihood), and prior probabilities (prior knowledge), to perform classification by assigning each object to the class with the maximum a posteriori probability. For $g$ groups, the Bayes rule assigns an object to the group $i$ when:

$$
P(i \mid \mathbf{x})>P(j \mid \mathbf{x}), \text { for } \forall j \neq i
$$

In this case, assuming the hypotheses of independence, we have for the random variables:

$$
P(i \mid \mathbf{x})=\frac{P(i) \prod_{k=1}^{n} P\left(x_{k} \mid i\right)}{\prod_{k=1}^{n} P\left(x_{k}\right)}
$$

where:

$$
P\left(x_{k} \mid i\right)=\frac{1}{\sqrt{2 \pi \sigma_{i k}^{2}}} e^{\frac{\left(x_{i}-\mu_{i k}\right)^{2}}{2 \sigma_{i k}^{2}}}
$$

being $P(\mathbf{x} \mid i)$ the probability of obtaining a particular set of features $\mathbf{x}$, given that the object belongs to the group $i$ and $P(i)$ is the a priori probability, i.e. the probability of choosing the group $i$ without known any feature of the object. 


\subsection{Database}

The experiments are performed over VisTex color textures database 17]. This database is maintained by the Vision and Modeling group at the MIT Media Lab. The full database contains images representative of real-world textures under practical conditions (lighting, perspective, etc.). In this work the 54 images of resolution $512 \times 512$ were split into 16 non-overlapping sub-images of $128 \times 128$. These images are available on de Outex site as test suite Contrib_TC_00006 [10].

\section{Results and Discussion}

In order to evaluate the quality of proposed method we set, based on work of [1], the $r_{\max }=20$, totaling 335 successive dilations. Additionally we make a uniform quantization of the image $I$ using a color map with 65536 colors. The source image $I$ is quantized by matching colors with the nearest color in the color map. This procedure aims decrease the number of color in source images.

The Table 1 1 shows the result for the proposed method in Vistex database. The $95.25 \%$ of accuracy demonstrates the high quality of the proposed method. This results use all features between $r=5$ and $r=20$, totaling $313 \log V(r)$ features. We do not use the first's radius because they not contain relevant features. It is due the quantization used, that separates the possible colors points on RGB color cube transform (i.e. the initial dilation of the points does not have any interaction with other points due the distance). More studies about the ideal quantization and ideal $r_{\max }$ parameter need be made in other databases. The high accuracy obtained impedes this research here, since several parameters will reach a high accuracy.

Obviously, this method needs be tested in more hard conditions, such different illuminations conditions and different acquisition devices. The RGB is a device-dependent color model, i.e. different devices detect or reproduce a given RGB value differently, since the color elements (such as phosphors or dyes) and their response to the individual $\mathrm{R}, \mathrm{G}$, and $\mathrm{B}$ levels vary from manufacturer to manufacturer, or even in the same device over time. Thus an RGB value does not define the same color across devices.

However, if this approach does not work with these difficulties, many alternatives to solve these problems are known. The use of color management systems are an alternative, but not always available. Apply this same methodology over other color spaces, such HSV, are another possibility.

\subsection{Comparison with Methods That Do Not Use Spacial Information}

In order to evaluate the quality of proposed method against another approaches, we compare it with 3 another methods of same class, i.e. who do not use information about spatial distribution of pixels to build the features. They are: 
Table 1. Comparison between methods that do not use the spatial distribution of gray levels as features

\begin{tabular}{cccc}
\hline \hline Method & No. of descriptors No. of images correctly Sucess rate \% \\
& \multicolumn{3}{c}{ classified } \\
\hline VFD RGB cube & $\mathbf{3 1 3}$ & $\mathbf{8 2 3}$ & $\mathbf{9 5 . 2 5}$ \\
Histogram ratio & uncertain & 484 & 56.02 \\
Chromaticity & 25 & 599 & 69.32 \\
First-order & 18 & 777 & 89.93 \\
\hline \hline
\end{tabular}

- Histogram ratio features [12]: this method utilizes an the 3-D xyY color histogram of a given image to calculate the self-relative histogram ratio features. The number of features varies from class to class since it depends on how many common histogram bins exist among each class.

- Chromaticity moments [11]: The method uses the CIE xy chromaticity diagram of an image and a corresponding set of two-dimensional and threedimensional moments to characterize a given color texture. We used the 5 T-type +5 D-type moments (CM55), totaling 25 features.

- First-order statistics of RGB channels [16]: Given the image, simple statistics as mean, variance, skewness, kurtosis, energy and entropy are calculated of each RGB channels, totaling 18 features.

The table 1 show the results. We can see the superior quality of the proposed approach, since the closest result is the first-order method, with 89.93 of accuracy. Is important to say that the work of [12] show an accuracy of $96.36 \%$ in Vistex database. However the confection of the database is another. He perform the experiments in a set of 164 color textures images of size $128 \times 128$, where he draws randomly from each image a subsample of $100 \times 100$. This result in a database where all samples of same class are very similar, unlike of de Vistex database used here. Due this, very bad results are reached by this method. The same problem occurs with [11] work.

\subsection{Comparison with Methods That Use Spatial Information}

The most methods of texture analysis use the spatial information directly (e.g. GLCM) or indirectly (e.g. Gabor filters) to build they features. We will compare our methodology with them too. The configuration used in these methods is presented below.

- Gabor filters [6] : is, basically, a bi-dimensional Gaussian function modulated with an oriented sinusoid. The convolution of the image with the family of Gabor filters in different scales and rotations produce the features. We use 64 filters (8 rotations and 8 scale filters) with lower and upper frequency equal to 0.01 and 0.4 , respectively. The individual parameter of each filter is defined by [8]. 
- Gray Level Co-occurrence Matrix (GLCM) [4]: they are the joint probability distributions between pairs of pixels at a determined distance and direction. For this comparison, distances of 1 and 2 pixels with angles of $-45^{\circ},-90^{\circ}$, $45^{\circ}, 90^{\circ}$ were used. Contrast, correlation, energy and homogeneity measures are computed from resulting matrices, totalizing a set of 32 descriptors. A non-symmetric version has been adopted in experiments.

The Table 2 shows the results. Despite the difference in methodologies, the $95.25 \%$ of accuracy, against $94.44 \%$ of Gabor filters and $92.47 \%$ of GLDM, is a very impressive result for a method that use only color information to build their characteristics. Moreover, since each method explores different texture characteristics, the use of our approach in conjunction with traditional methods is quite possible. The Gabor filters or GLDM, for example, uses the spatial distribution of pixels to characterize the texture and, the proposed methodology explores, basically, the color distribution, they are complementary information.

For all results (except histogram ratio that use classification scheme of [12]), we use LDA analysis in a 10-fold cross-validation. We summarize the original features into principal components that represent $99.99 \%$ of total variance explained. The new features, also called canonical features, are then used in the Bayesian classifier. Thus, although our approach has the largest number of feature between all tested methods, the number of canonical features obtained after dimensionality reduction with LDA is very similar.

Table 2. Comparison between proposed method and traditionally methods that use spatial information

\begin{tabular}{cccc}
\hline \hline Method & No. of descriptors No. of images correctly Sucess rate \% \\
& \multicolumn{3}{c}{ classified } \\
\hline \hline VFD RGB cube & $\mathbf{3 1 3}$ & $\mathbf{8 2 3}$ & $\mathbf{9 5 . 2 5}$ \\
GLCM & 32 & 799 & 92.47 \\
Gabor filter & 64 & 816 & 94.44 \\
\hline \hline
\end{tabular}

\section{Conclusion}

A simple and efficient method for color texture classification has been presented. The called RGB color cube transform map the existing colors of texture in a cube, and the volumetric fractal dimension uses these color distribution information to build the features.

A comparison with several methods are performed and, although do not use any type spatial information the proposed method achieves high classification rates, comparable, if not better, with traditional texture analysis methods. Further research will investigate the ideal quantization and the optimal $r_{\max }$ parameter, and will also examine the performance in other databases with different illumination sources and acquisition devices. 


\section{References}

1. Backes, A.R., Casanova, D., Bruno, O.M.: Plant leaf identification based on volumetric fractal dimension. International Journal of Pattern Recognition and Artificial Intelligence 23(6), 1145-1160 (2009)

2. Chellappa, R., Chatterjee, S.: Classification of textures using gaussian garkov random fields. IEEE Transactions on Acoustics, Speech, and Signal Processing 33(4), 959-963 (1985)

3. Fabbri, R., da F. Costa, L., Torelli, J.C., Bruno, O.M.: 2D euclidean distance transform algorithms: A comparative survey. ACM Computing Surveys 40(1), 1-44 (2008)

4. Haralick, R.M.: Statistical and structural approaches to texture. Proceedings of IEEE 67(5), 786-804 (1979)

5. Hirata, T.: A unified linear-time algorithm for computing distance maps. Information Processing Letters 58, 129-133 (1996)

6. Jain, A.K., Farrokhnia, F.: Unsupervised texture segmentation using gabor filters. Pattern Recognition 24(12), 1167-1186 (1991)

7. Keller, J.M., Chen, S., Crownover, R.M.: Texture description and segmentation through fractal geometry. Computer Vision, Graphics, and Image Processing 45(2), 150-166 (1989)

8. Manjunath, B.S., Ma, W.-Y.: Texture features for browsing and retrieval of image data. IEEE Transactions on Pattern Analysis and Machine Intelligence 18(8), 837-842 (1996)

9. Meijster, A., Roerdink, J.B.T.M., Hesselink, W.H.: A general algorithm for computing distance transforms in linear time. In: Proceedings of the 5th International Conference on Mathematical Morphology and its Applications to Image and Signal Processing, pp. 331-340 (2000)

10. Ojala, T., Mäenpää, T., Pietikäinen, M., Viertola, J., Kyllönen, J., Huovinen, S.: Outex - new framework for empirical evaluation of texture analysis algorithms. In: Proceedings 16th International Conference on Pattern Recognition, pp. 701-706 (2002)

11. Paschos, G.: Fast color texture recognition using chromaticity moments. Pattern Recognition Letters 21(9), 837-841 (2000)

12. Paschos, G., Petrou, M.: Histogram ratio features for color texture classification. Pattern Recognition Letters 24(1), 309-314 (2003)

13. Randen, T., Husøy, J.H.: Filtering for texture classification: A comparative study. IEEE Transactions on Pattern Analysis and Machine Intelligence 21(4), 291-310 (1999)

14. Tricot, C.: Curves and Fractal Dimension. Springer (1995)

15. Tuceryan, M., Jain, A.K.: Texture segmentation using voronoi polygons. IEEE Transactions on Pattern Analysis and Machine Intelligence 12(2), 211-216 (1990)

16. Tuceryan, M., Jain, A.K.: Texture analysis. In: Chen, C.H., Pau, L.F., Wang, P.S.P. (eds.) The Handbook of Pattern Recognition and Computer Vision, pp. 207-248. World Scientific (1998)

17. VisTex. Vision texture database (2009), http://vismod.media.mit.edu/vismod/imagery/VisionTexture/vistex.html

18. Weszka, J.S., Dyer, C.R., Rosenfeld, A.: A comparative study of texture measures for terrain classification. IEEE Transactions on Systems, Man, and Cybernetics 6(4), 269-285 (1976) 